\title{
Understanding Decision Making through Complexity in Professional Networks
}

\author{
Kon Shing Kenneth Chung \\ Complex Systems Research Group, Project Management Program, The University of Sydney, Camperdown, NSW 2006, Australia \\ Correspondence should be addressed to Kon Shing Kenneth Chung; ken.chung@sydney.edu.au
}

Received 29 May 2014; Accepted 13 November 2014; Published 11 December 2014

Academic Editor: Mahyar A. Amouzegar

Copyright (C) 2014 Kon Shing Kenneth Chung. This is an open access article distributed under the Creative Commons Attribution License, which permits unrestricted use, distribution, and reproduction in any medium, provided the original work is properly cited.

\begin{abstract}
The attitudes of general practitioners (GP) play an influential role in their decision making about patient treatment and care. Considering the GP-patient encounter as a complex system, the interactions between the GP and their personal network of peers give rise to "aggregate complexity," which in turn influences the GP's decisions about patient treatment. This study models aggregate complexity and its influence in decision making in primary care through the use of social network metrics. Professional network and attitudinal data on decision making responsibility from 107 rural GPs were analysed. Social network measures of "density" and "inclusiveness" were used for computing the "interrelatedness" of components within such a "complex system." The "number of components" and "degree of interrelatedness" were used to determine the complexity profiles, which was then used to associate with responsibility in decision making for each GP. GPs in simple profiles (i.e., with low components and interactions) in contrast to those in nonsimple profiles, indicate a higher responsibility for the decisions they make in medical care. This study suggests that social networks-based complexity profiles are useful for understanding decision making in primary care as it accounts for the role of influence through the professional networks of GPs.
\end{abstract}

\section{Introduction}

The attitudes of health professionals such as general practitioners (GPs) play an influential role in their delivery of medical care, such as communication and decision making in patient care. A number of other studies have focused on examining the GP's delivery of medical care from various perspectives: quality of the consultation [1]; psychological and medical functions [2]; measuring informed decision making through evaluating patient-GP encounters [3]; influence through professional networks $[4,5]$. More recently, there is evidence showing how medical care delivery can be conceptually analysed using principles of complexity thinking, where the GP-patient consultation is deemed to be a complex adaptive system [6].

According to Plsek and Wilson [7], "complexity thinking suggests that relationships between parts (of a system) are more important than the parts themselves, and that minimum specifications yield more creativity than detailed plans." It is useful to consider the meaning of systems within which complexity is situated and derived and the type of systems that is referred to in this paper. Broadly speaking, "systems" consist of multiple parts (or components) that are connected and interrelated in one or many ways [8]. An important principle of systems thinking is that a system is more than the sum of its parts. In social systems, for instance, the patient and GP may be considered agents (or components) where their interactions and dependence on each other constitute interrelatedness. Coupled with the agents' ability to rationalize and perform multiple actions, the interrelatedness helps to evolve a pattern of behaviour, for instance, during the consultation process that is situated within the larger healthcare system. It is the degree and uniqueness of such interrelatedness within and out of these systems that create complexity in an aggregate sense.

In this study, complexity in social systems through "network thinking" and how it impacts responsibility for decision making in medical practice are considered. By "social system," the study specifically refers to the collection of individual agents (e.g., general practitioners) and their interactions with 
other agents within the social system (e.g., peers, practice managers, nurses, etc.) as part of the larger healthcare system. While there are numerous studies in healthcare that have incorporated applied complex systems thinking $[6,9]$, most of them remain descriptive or conceptual at best and provide theoretical accounts or frameworks of how to study complexity [10]. Therefore, the motivating questions for this research are as follows. (i) Is there a theoretical basis for envisioning and studying complexity on an aggregate level (i.e., holistically) and with empirical evidence? (ii) Can the application of network science and robust measures in social networks analysis allow one to develop complexity profiles on an individual basis? (iii) How can these complexity profiles be utilized for understanding associations with individual outcomes such as attitudes to responsibility for decision making in medical care? In this paper, it is argued that, at the operational level, it is possible to characterize complexity based on the number of agents and degree of interaction between these agents within the social system. In particular, it is demonstrated using the professional networks of GPs how a complexity profile may be computed for each GP and how this complexity profile can then be associated with their responsibility for decision making in the GP-patient encounter. Measured using validated and reliable attitudinal statements from the GPs themselves, the decision-making variable allows one to understand the extent to which the GP or the patient is involved and responsible for decision making regarding health care, plans, and pathways. Thus, the study does not include any patient data about decision making nor does it capture patient networks as it models the personal and professional networks of GPs and their world view of the decision making involvement and responsibility during the GP-patient encounter.

The subsequent section on "Conceptual Foundations" contains a brief literature review of systems thinking and complexity science and demonstrates how social systems may be conceived as social networks, which, in turn, allows us to develop a model for capturing subconstructs of complex social systems such as number of components and degree of interactions or interrelatedness. In the third section discussion of the methodology includes details of how data were collected, the sample size, and instrument development process and also provides detailed information of attribute, relational data, and relevant measures. Finally, results of the analysis and discussion of the findings in light of theory follow prior to limitations, future work, and conclusion of the study.

\section{Conceptual Foundations}

Studies in complexity have been well documented in areas of physics, biology, artificial intelligence, computational science, and economics [11]. Although there are numerous definitions (and debates) of the term "complexity," the science of complexity usually deals with the "study of phenomena which emerge from a collection of interacting objects" [12]. There are currently a number of studies that have either explored issues surrounding complexity in health care $[10,13]$ or have applied principles of complex systems to the health care environment as a new lens for studying health care from a holistic perspective [14-16]. For instance, Nugus et al. [17] argue that the "continuity of care" model with respect to the patient's journey in the healthcare system is generally a linear one and current models do not accurately explain the efficiency of integrated care. In order to remedy this gap, they deployed ethnographic techniques and the complex systems approach to observe the interactions between the departments and units outside the emergency departments of two hospitals based in Sydney, Australia, arguing that their approach allowed explaining the interdependencies in a more holistic manner. The importance of this work demonstrates that the healthcare journey that encompasses decision making is not a linear one; it is rather crucial to account for the interactions, interdependencies, and interrelatedness of resources (human, physical, technological, etc.) in order to understand efficiency of the healthcare journey, including decision making by healthcare professionals. Bar-Yam et al. [18], on the other hand, adopt complex systems approach to suggest how the US health care system can achieve a taskresource fit while optimising on cost and resource constraints. At a microlevel, in general practice particularly, Innes et al. [6], by using individual patient consultation as their unit of analysis, suggest that the GP-patient consultation can be considered a complex system. This is because of several features that complex systems exhibit that are similar to the GP-patient encounter-for instance, the consultation process involves nonlinearity such that it leads to uncertainty and there is adaptation to the influence of outside agencies. In order to illustrate this, Innes et al. [6] use the example of the "hypertensive patient" in two scenarios of consultationone where the doctor focuses on a somewhat rigid pattern of consultation style and the other being the doctor adopting a natural and conversational style of consultation. In the first scenario, the doctor focuses on symptoms, medication problems, and attention to cardiovascular risk analysis. Here, the process is almost standard in that the pattern is linear and repeated with other patients. This may prevent novelty and the emergence of new information which may be useful for diagnosis. In the second scenario, the consultation conversation is rather free-flowing and this allows the "expression of novelty and creativity and, perhaps, a glimpse of surprising insights that may lead the consultation into completely new areas," thereby highlighting the nonlinearity characteristics of the consultation process.

In light of the current literature on complexity in health care, most studies are descriptive and are mostly qualitative in nature. While these studies provide rich narrative accounts of the complex system, it does not provide validated and reliable metrics for envisioning and studying complexity in health care contexts. It is therefore useful to consider a rational approach for studying complexity in health care settings.

Although the definition of complexity is debatable and context dependent $[11,19]$, for the purpose of this study, complexity is defined in terms of one of the most salient concepts postulated by "aggregate complexity" [20]-the interrelatedness of components of a system [10]. According to Kannampallil et al. [10], complexity of a system is relative 


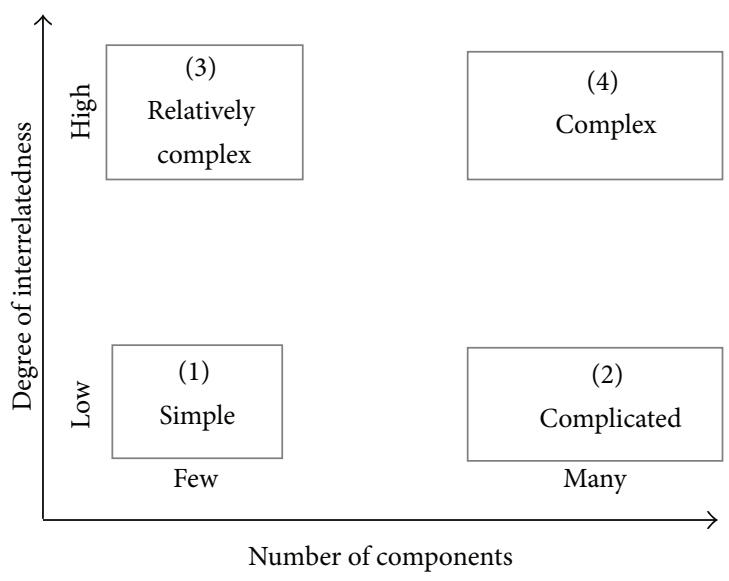

FIGURE 1: Range of complexity [10].

in the sense that complexity is a function of the number of components (NoC) and the degree of interrelatedness (DoI) within the system. This definition is in congruence with others in the field $[9,11,12,20]$. In other words, as both variables increase, so does complexity of the system. It is also important to note that while increasing the number of components may make the system "complicated," it is the degree of interrelatedness or, in other words, the unique relationships (both manifest and latent) that make the system "complex." As a consequence, the interrelatedness of system components results in properties that characterise complex systems [9], these properties being nondecomposability (that systems cannot be understood by focusing on components in isolation), emergence (where unexpected behaviour arises as a result of component interactions), nonlinear behaviour (characterised as nonpredictability and nonproportionality of behaviour), and self-organisation (where individual actors take on different structural positions so the system can be maintained). Accordingly, by combining ranges of extremes for both variables, there can be four conditions (although not postulated in a prescriptive or exhaustive manner) to characterise the range of complexity as shown in Figure 1.

Firstly, there are simple systems with few components and low interrelatedness (1), whereby the system along with its behaviour is easily predictable and is easily understood, managed, and described. For instance, an individual GP who runs his own practice by himself may only have few components, such as patients, notes and computer, and relations (interaction with computer, patient, and stationery). The GP is considered to be in a very simple system. Secondly, systems with many components and low interrelatedness (2) are also, in many cases, quite predictable to a certain extent because of the low interrelatedness, for instance, a receptionist in a general practice who handles many phone requests and relies only on the computer booking system. Thirdly, relatively complex systems have few components but a high degree of interrelatedness (3). Such systems can be studied as a "whole" because of their few components but high level of interrelatedness-for example, section of an emergency department where members are few but the interactions are quite diverse. Finally, complex systems are systems exhibiting high degree of interrelatedness and many components (4), for example, multiple critical care teams attending to multiple patients in a disaster-struck area.

Although the framework proposed above is useful for profiling complexity, it is still unclear what is meant by "degree of interrelatedness." To this end, the paper draws upon principles from the study and analysis of social networks and the network-thinking paradigm.

2.1. Network Thinking. If the heart of aggregate complexity lies in the relationship between components in an attempt to access the holism and synergy resulting from it, then it is useful to consider some classical studies in evaluating the structure of such relationships. Drawing on studies in social network analysis [21] and the "network paradigm" [22], it is contended that "network thinking" serves as a useful theoretical and analytical lens for studying complex networks. It is argued that network thinking, social network analysis in particular, is a useful methodology for the operationalization of the construct of aggregate complexity. A social network, simply, is a collection of actors (e.g., individuals or teams) and ties (e.g., relationships such as "seek advice from" and "are friends with") that bind the actors. While, for social network scholars, the raison d'être is that the structure of relationships among actors has important behavioral, perceptual, and attitudinal consequences both for the individual units and for the system as a whole [23], the tenet of most complex system thinkers is that "it is in the structure of the system, not the effort of the people in it, that determines the outcome.... The way to gain more influence is to understand the structure of the system" [24]. It is argued that literature in social networks is therefore a useful theoretical basis and analytical methodology for studying complexity in healthcare $[25,26]$.

\subsection{Computing Interrelatedness Using Network Metrics.} Drawing on closing remarks from Kannampallil et al. [10], “...complex systems can typically be considered in terms of functionally smaller components and the relations between them, based on theoretical, rational, and practical considerations.... There often is a structure in the relationships that exist between care providers, artifacts, and patients.... As such, it is possible to characterize it as a network of actors, where (at a high level of decomposition) the nodes are actors (or artifacts) and the edges are their relationships." Although no single operational definition of the construct, interrelatedness, is offered, it is argued that there are two salient measures in social networks analysis that might help develop an operational definition of the construct.

A lay implication of the term "interrelatedness" suggests either to relate or to connect to one another. In social network parlance, there are a number of validated and reliable measures that are used to describe exactly how well connected a certain network is. The degree of interconnectedness within networks, also named cohesion or closure, generally referred to as network density, is important for predicting the level of homogeneity among actors [27]. Density is measured as the ratio of existing ties to the maximum possible ties [28]. 
A density value of 1 indicates that everyone is connected to everyone else within the network (i.e., a clique structure) whereas a value of 0 indicates a very sparse network. According to Granovetter [29], due to the homophily principle in social networks where actors with similar attributes and interest tend to connect with each other, information travels at high velocity and becomes redundant rapidly because of high density within the network. This possibly explains why the diffusion of innovation and adoption of a new drug were faster for doctors with denser social connections than those without [5]. High density in a social network is thus characterized by the frequent exchange of information, knowledge, and other resources among individuals. Cohesive groups are thus likely to be conducive to mechanisms of social influence and contagion [30].

A related social network measure of interconnectedness is the notion of "inclusiveness." Inclusiveness refers to the number of connected actors within the social network. In other words, within a social network, it is the total number of actors minus the number of isolated actors [31]. So if one considers a network of 10 actors, with 5 isolated actors, inclusiveness would be 5 . However, in order to allow for standardization and comparison across several networks (similar to the density measure), it is useful to express inclusiveness as a proportion of the total number of actors within the network. Therefore, using the example above, inclusiveness expressed as a proportion of the entire network would be 0.5 , with the range being 0 to 1 . Therefore, while inclusiveness represents the connectedness of individual actors within a network, density captures the extent to which the connections are current as compared to the latent. So while inclusiveness is a measure based at the actor level, density is about the extent to which the actors are connected and is situated at the tie level.

The notion of inclusiveness is a useful indicator of social network membership as well as group dynamics. Mitchell and Trickett [32], in their work to understand determinants of social networks, offer a comprehensive list of operational definitions of social network membership ordered by inclusiveness. For instance, one may ask the question "Name all the people who are important to your work or whom you are close to in the current practice" to qualify for inclusiveness. Pfeil and Zaphiris [33] in their study of online discussion forums for older people associated network inclusiveness with the types of discussions that took place. It was found that emotional communication was linked to stronger inclusiveness scores compared to factual communication. Interestingly, they also found that emotional communication was associated with density, unlike factual communication, which had weaker connections between individual members within the network. Although the measures of density and inclusiveness are different per se, conceptually, they are positively associated and are thus useful to consider in conjunction as a valid measure of interrelatedness.

2.3. Complexity Profiles in Context of General Practitioners. Gask and Usherwood [2] claim that there are three functions in a medical consultation, namely, Building the
Relationship; Collecting Data; and Agreeing on a Management Plan. The first stage entails the GP building rapport with the patient through warm greeting, active listening, and detecting and responding to emotional issues. The second stage involves noninterruption in the process of eliciting the patient's explanatory model in order to develop a shared understanding of the problem. It is quite obvious that the Collecting Data stage would involve interactions with not only the patient but also both human (e.g., peers and specialists) and nonhuman (e.g., computer database and special devices) resources if necessary. The third stage involves functions where the GP reassures and advises the patient on medical plans or prescriptions. This stage involves mutuality in that the GP and the patient have the opportunity to discuss how much the patient wishes to be involved in making decisions in the consultation. For instance, the compliance of patients, especially when receiving long-term treatment, is enhanced when patients have the opportunity to partake in decisions about treatment $[34,35]$. In a recent study of rural clinician's perception of shared decision making and decision aid by King et al. [36], it was found that $69 \%$ (of the 173 physicians sampled) agreed that the patient and the GP "should decide" together on clinical decisions, whereas only $42 \%$ perceived that it was "actually done" in reality.

Based on the "range of complexity" framework [10] discussed earlier, it is proposed that interrelatedness and density are potentially useful measures of interrelatedness, which can then be used in conjunction with the number of components to characterize complexity for GPs. Furthermore, it is postulated that GPs in the "simple" complexity profile, because of their lower number of components and degree of interrelatedness, would have more opportunities in discussing patients' treatment plans and encourage patients to partake in the medical decision making process. In a study of the effects of patient, GPs, and healthcare system characteristics on specialty referral decision making [37], it was found that patient characteristics (such as clinical cues presented and level of medical insurance) accounted for the largest effect. This suggests that, in general, decisions made took place mainly because of the interaction with the patient and not necessarily as a result of navigating (i.e., interacting) through the GP's professional network. In other words, since the interaction generally took place only between the GP and the patient, there were low numbers of components and interactions (i.e., simple profile). It is also argued that as the number of components increases and the degree of interrelatedness increases during general practice, overall complexity within the social system increases. The increase in complexity based on interdependence and interconnectedness of human and nonhuman resources adds to the overall cognitive, taskrelated and contextual load, and is therefore detrimental to performance $[38,39]$. As it stands, GPs, and particularly rural GPs, are heavily burdened in terms of workload [40, 41]. So, if a GP requires a piece of information but has to navigate through at least three or four resources in order to obtain it, as compared to a GP who can obtain that information through first contact with the resource within their network, intuitively, the latter's network is simpler than the first in terms of profiling complexity. In social networks 
literature, there is plenty of evidence to suggest that while inclusiveness and density are robust measures for interactivity, engagement, and interconnectedness, highly dense networks (e.g., cliques) are generally closed networks (like closed systems) where everyone is connected to everyone and also results in information overload and redundancy, which is not conducive to performance [42]. Based on these arguments above, the following is hypothesised.

H1: GPs in the "simple" complexity profile account for higher responsibility for decision making than those in the "complex" profile.

H2: GPs in the "simple" complexity profile account for higher responsibility for decision making than those in the "relatively complex" profile.

H3: GPs in the "simple" complexity profile account for higher responsibility for decision making than those in the "complicated" profile.

In summary, the crux of $\mathrm{H} 1$ to $\mathrm{H} 3$ suggests that as GPs become more interdependent in terms of their professional network for seeking advice (i.e., as they move from simple to complicated to complex), the extent to which they are involved or take responsibility for their decision making (i.e., this transfers mostly to the patient) becomes lower than those GPs who are less interdependent on their professional network. As stated earlier, a highly interdependent network adds to the overall cognitive, task-related, and contextual load of the GP consultation, which has been linked to detrimental performance.

\section{Methodology}

The chosen context of the study is the rural general practitioners (GPs) of New South Wales, Australia. The work of rural GPs is knowledge intensive because of the nature of their work, extensive medical expertise, high patient to GP ratio, long work hours, usage of advanced medical technologies, provision of diverse healthcare services, and so on [43]. GPs working in rural areas are geographically more occupationally isolated from populated practices. Furthermore, rural GPs often carry out procedures in situations with limited resources or personnel and are implicitly required to adapt to protocols and codes of conduct of rural settings [44]. Such problems and others such as decreasing performance as GPs age, lack of association with professional peers, obsolescence with modern technology, and isolation from community not only hinder performance but also make this study potentially interesting and practically useful [45].

The dataset used for this study was obtained from a previous research project where both attribute-level data (such as organizational fellowships (e.g., Royal Australian College of General Practitioners), number of years practiced, country, and graduation from medical school) and social network data of rural GPs were collected [46]. The dataset constitutes responses from 110 rural GPs collected from a reliable and validated survey instrument administered to all the 17 divisions of rural general practice in NSW Australia.
The egocentric approach for collecting network data was adopted for the study because of its practicality and feasibility. In this approach, the actor of interest is referred to as the "ego" and the actors, referred to by the "ego" as their affiliates, advisors, friends, or relatives, are known as "alters" [31]. Name generators are used in order to elicit alters' names. The following name generator was used to elicit names from a GP's professional network.

"By 'professional network', we mean professional
people whom you associate, interact or work with
for the provision of care to patients (e.g., nurses,
admin staff, specialists, pathologists, doctors, etc.)
Looking back over the last six months, please
identify people (up to 15 maximum) who are
important in providing you with information or
advice for providing care to patients."

Name interpreter questions are also commonly asked to elicit some attribute data about the alters and ties. In this case, it was requested for GPs to indicate the occupational code (e.g., nurse, practice manager, specialist, etc.) as well as the geographical location (e.g., same practice, other practice, etc.) of each alter. The strength of each tie, measured by "how long they knew each other," "frequency of interaction," "type of relationship," and "degree of closeness" [47], was also solicited.

To determine the relationship between elicited alters in order to complete the network structure, GPs were asked to determine how the members of their professional network relate to each other based on a five-point degree of closeness scale ranging from "especially close" to "do not know each other." That is, for each nominated alter, the GP would determine a closeness scale for every other alter. Although this approach has been criticized in the past for its recall reliability and accuracy [48], later studies confirmed that people also remembered long-term or typical patterns of interaction with other people rather well [49]. Furthermore, the free recall method elicits richer data on the social networks of people whereas the fixed choice method influences people to elicit accurate information on the most important relationships (i.e., strong ties) [50].

\subsection{Measures}

3.1.1. Responsibility for Decision Making. Two question items based on a validated and reliable questionnaire by Cockburn et al. [34] for assessing perceived attitudes to responsibility for decision making were used. Based on 7-point Likert scales (strongly disagree to strongly agree), the items were as follows.

\section{"The majority of patients do not wish to be involved in decision making about their treat- ment" and \\ "most patients would prefer the doctor to take responsibility for their medical problems."}

A composite score was then calculated to give an overall score for attitudes to responsibility for decision making. 
Higher scores thus indicate that the GP feels positively about patients being involved in making decisions in the consultations.

3.1.2. Density. Density is a measure of network cohesiveness and is the ratio of existing number of ties to the maximum possible ties. For an undirected network with $n$ actors, density $D$ is defined as

$$
D=\frac{\sum_{i, j=1}^{n} x_{i j}}{n(n-1) / 2}
$$

where $x_{i j}$ is the value of the connection from $i$ to $j$. For this study, ego-alter ties were not considered but only alteralter ties in the density calculation were considered, as is appropriate with ego network density [51].

3.1.3. Inclusiveness. Inclusiveness measures the proportion of actors that are connected to the total number of actors within the network. Inclusiveness $I$ is defined as

$$
I=\frac{\sum_{i} x_{i}}{g},
$$

where $g$ is the total number of actors and $x_{i}$ is 1 when there is a tie coming from or going to node $I$ and 0 otherwise. Again, in the calculation of inclusiveness, ego-alter ties were not considered but only alter-alter ties were considered because of the nature of ego network data collection approach (i.e., if ego-alter ties were considered, inclusiveness for all GPs will always result in 1).

3.1.4. Interrelatedness. Interrelatedness is the product of $D * I$ where $D$ is density and $I$ is inclusiveness.

\section{Results}

Results from the respondents surveyed indicate that the typical rural GP has been in rural practice for 20.24, with 13.63 years in the current practice (Table 1). Although there are solo practices (a one-doctor-only practice), the typical rural GP, as indicated by the results, works with at least 4 colleague GPs in the same practice. Furthermore, 90 (or 81.8\%) are male and 20 (or $18.2 \%$ ) are female, and 85 (or $77.3 \%$ ) have hospital appointments.

In order to construct the complexity profiles, the mean of interrelatedness (0.5739) and the mean of "number of components" (8.78) were used as cut-points on the $y$-axis and $x$-axis, respectively. As a result, $29.9 \%$ of GPs belong to the complex profile, $28 \%$ to the relatively complex, $16.8 \%$ to the complicated profile, and $25.2 \%$ to the simple profile. Table 2 shows the breakdown in terms of the number of GPs with percentages for each complexity profile followed by the scatterplot Figure 2.

4.1. Hypothesis Testing. The independent samples $t$-test was used to test hypotheses H1, H2, and H3. This particular statistical test evaluates whether there is a statistically significant
TABLE 1: Descriptive statistics.

\begin{tabular}{lcccc}
\hline & Min. & Max. & Mean & Std. dev. \\
\hline Years in rural practice & 1 & 50 & 20.24 & 10.44 \\
Years in current practice & 1 & 43 & 13.63 & 10.37 \\
Number of GPs in current practice & 0 & 25 & 4.54 & 4.11 \\
Responsibility for decisions & 2 & 13 & 7.21 & 2.59 \\
Number of components & 1.00 & 15.00 & 8.78 & 3.97 \\
Inclusiveness & 0.00 & 1.00 & 0.83 & 0.31 \\
Density & 0.00 & 1.00 & 0.58 & 0.37 \\
Interrelatedness & 0.00 & 1.00 & 0.57 & 0.37 \\
\hline
\end{tabular}

TABLE 2: Distribution of GPs by complexity profiles.

\begin{tabular}{lcc}
\hline Profiles & Frequency & Percentage \\
\hline Simple & 27 & $25.2 \%$ \\
Complicated & 18 & $16.8 \%$ \\
Relatively complex & 30 & $28.0 \%$ \\
Complex & 32 & $29.9 \%$ \\
Total & $\mathbf{1 0 7}$ & $\mathbf{1 0 0 \%}$ \\
Missing & 2 & \\
\hline
\end{tabular}

difference in the mean scores for the two groups (e.g., of GPs in simple and complex profiles). Statistically, it tests the probability that the two sets of scores came from the same population.

For $\mathrm{H} 1$, the $t$-test results suggest that there is a statistical difference in the overall attitude scores for responsibility for decisions for GPs in the "simple" complexity profile $(M=8.37, \mathrm{SD}=2.72)$ and GPs in the "complex" profile $(M=6.31, \mathrm{SD}=2.07) ; t(57)=3.29, P=0.002$ (twotailed). The magnitude of the differences in the means (mean difference $=2.06,95 \% \mathrm{CI}: 0.81$ to 3.31 ) was large (eta squared $=0.16$ ). Furthermore, it is evident that the mean attitude score for responsibility for decisions of GPs in the "simple" complexity profile is higher than that of GPs in the "complex" profile. Therefore, regarding $\mathrm{H} 1$, there is sufficient evidence to reject the null hypothesis and therefore support the alternate hypothesis. One may thus infer that GPs in the "simple" complexity profile score higher in attitude to responsibility for decisions compared to GPs in the "complex" profile.

For $\mathrm{H} 2$, the $t$-test results showed that there is no statistical difference in the overall attitude scores for responsibility for decisions for GPs in the "simple" complexity profile $(M=$ $8.37, \mathrm{SD}=2.72$ ) and GPs in the "relatively complex" profile $(M=7.73, \mathrm{SD}=2.42) ; t(55)=0.936 ; P=0.353$ (twotailed). Although the mean attitude score for responsibility for decisions of GPs in the "simple" complexity profile is higher than that of GPs with the "complex" profile, there is no statistical significance. Therefore, for $\mathrm{H} 2$, there is no sufficient evidence to reject the null hypothesis and therefore there is no evidence to support the alternate hypothesis. Thus, one cannot infer that GPs in the "simple" complexity profile score higher in attitude to responsibility for decisions compared to GPs in the "relatively complex" profile.

For $\mathrm{H} 3$, the $t$-test results suggest that there is a statistical difference in the overall attitude scores for responsibility for 


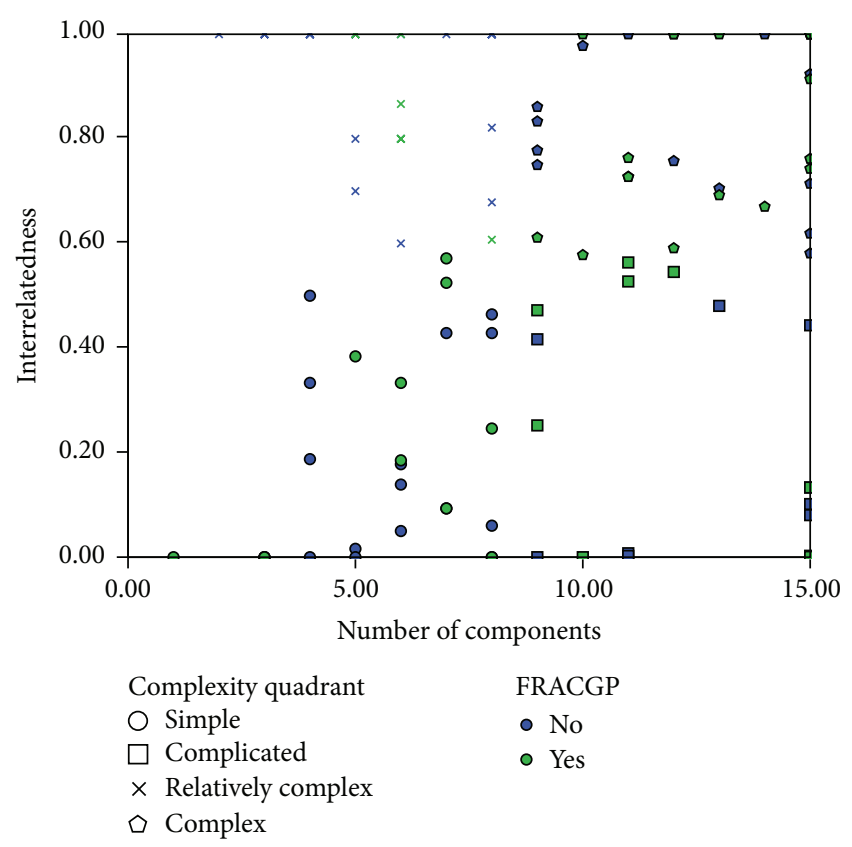

FIGURE 2: Scatterplot showing the complexity profiles.

decisions for GPs in the "simple" complexity profile $(M=$ $8.37, \mathrm{SD}=2.72)$ and GPs in the "complicated" profile $(M=$ $6.22, \mathrm{SD}=2.82) ; t(43)=2.56 ; P=0.014$ (two-tailed). The magnitude of the differences in the means (mean difference $=$ 2.15, 95\% CI: 0.45 to 3.84 ) was large (eta squared $=0.13$ ). Furthermore, it is evident that the mean attitude score for responsibility for decisions of GPs in the "simple" complexity profile is higher than that of GPs with the "complicated" profile. Therefore, for $\mathrm{H} 3$, there is sufficient evidence to reject the null hypothesis and therefore support the alternate hypothesis. One may thus infer that GPs in the "simple" complexity profile score higher in attitude to responsibility for decisions compared to GPs in the "complicated" profile.

\section{Discussion}

Based on the results, it is clear that the mean score for attitudes to responsibility for decisions for GPs in the "simple" profile is higher than the mean scores of those GPs in the "complex" (H1), "relatively complex" (H2), and "complicated" (H3) profiles. However, statistical significance was found only for $\mathrm{H} 1$ and $\mathrm{H} 3$. The results are interesting in that they are indicative of several aspects of aggregate complexity.

(1) Simple versus Complex. When it comes to GPs in simple profile versus GPs in complex profile, it is obvious that the interplay between the number of components and degree of interrelatedness in each of the profiles is associated with attitudes to responsibility for medical decisions. GPs with a larger number of social contacts and a higher degree of interrelatedness within their network tend to score lower in attitudes to responsibility for medical decisions (i.e., they are less involved or responsible) than GPs with fewer number of social contacts and a lower degree of interrelatedness.

(2) Simple versus Relatively Complex. GPs in the simple profile also score higher in terms of overall attitudes to responsibility for medical decisions than GPs in the relatively complex profile. GPs in the latter quadrants have networks that are characterized by a high degree of interrelatedness and lower number of components as compared to the simple profile. However, this finding is not statistically significant and claiming this hypothesis to be valid would be spurious.

(3) Simple versus Complicated. This finding is interesting in that although aggregate complexity is defined as the interrelatedness of the components within a system, it is found that, by increasing the number of components alone, GPs with a low number of components and low degree of interrelatedness score higher in attitudes to responsibility for medical decisions as compared to GPs who have a higher number of components and low degree of interrelatedness. This finding is statistically significant with a large effect size as well.

Considering hypotheses $\mathrm{H} 1-\mathrm{H} 3$ altogether, there seems to be a pattern where as complexity increases (as defined by the profiles) as contrasted using simple-complicated, simplerelatively complex, and simple-complex profiles, GPs in those roles tend to score lower in attitudes to responsibility for medical decisions. This means that as the profile changes from simple to nonsimple one, the extent to which GPs want patients to be involved in decision making in the consultation diminishes. Although a linear relationship is not suggested, it is interesting to question why this is so.

The fact that the varying complexity of professional networks of the GPs is associated with their attitudes to responsibility for medical decision making tells a different side of the story. Without delving into the details of the interactions (e.g., content and nature of the communication) and the specific contexts within which it took place (e.g., was the GP seeking advice from these close contacts while in hospital settings and not their usual practice setting?), it is useful to consider from a structural and relational perspective.

Possible explanations for this paper's findings can be drawn from studies in social networks literature. Intuitively, it is reasonable to argue that as complexity profiles evolve from simple to complex, there is a point when performance starts to decline because of the high degree of interrelatedness. Consider, for instance, a knowledge-intensive scenario where a complicated surgery is taking place involving three surgeons and another two are added later. With three surgeons, the maximum number of directed communication flows can be derived using the formula $n *(n-1)$ or $3 *(3-$ $1)=6$. Adding a further two more surgeons increases the potential maximum number of communication flows to $20(5 *(5-1))$, thereby increasing the interrelatedness within the system. Given the results, one may suggest that GPs in simple network structures have low degree of interrelatedness and are most likely comfortable enough in 
terms of workload efficiency and possibly time, in order to allow them to discuss with patients regarding treatment decisions. GPs in nonsimple (i.e., complicated or complex) networks, on the other hand, are highly interrelated and interdependent on their relational sources (and potentially nonrelational ones such as the Internet or online medical databases) to the extent that they do not believe in involving their patients in the treatment/medical decision process compared to GPs in simple networks. Furthermore, Burt [52] claims that increasing network size (number of direct contacts or components) without considering the diversity reached by the contacts makes the network inefficient in many ways. Therefore, the number of nonredundant contacts is important for performance to the extent that redundant contacts would lead to the same people and hence provide the same information benefits. It is definitely plausible, then, that nonredundancy is in play when comparing GPs in "simple" complexity profiles to GPs in other complexity profiles. In line with Burt's argument, this also possibly explains why potential performance (measured by attitudes to care in responsibility for decision making) is affected.

\section{Conclusion}

In this study, an aggregate complexity framework (ACF) based on social network measures of density and inclusiveness was developed for understanding how complexity profiles of one's social network can impact one's attitudes towards medical practice. Aggregate complexity deals with how individual elements (within a system, e.g., social system) work harmoniously and holistically to create systems with complex behaviour. Representing social systems as social networks, it was proposed that, by studying the number of actors and the degree of interrelatedness within one's professional network, it is possible to characterise one's network with a complexity profile ranging from "simple," "complicated," and "relatively complex" to "complex." Results from a sample dataset of 107 rural general practitioners (GPs) in New South Wales, Australia, show that GPs with a "simple" profile score higher on attitudes to responsibility for medical decisions compared to those with "nonsimple" profiles.

The contribution and merit of this paper are threefold: (i) at the theoretical level, a framework for understanding aggregate complexity in terms of the degree of interrelatedness within professional networks of medical practitioners is proposed; (ii) methodologically, the paper provides a validated and reliable survey instrument that can be used to operationalize the ACF in other healthcare settings (e.g., cancer care coordination); and (iii) at the domain-level, it was demonstrated how understanding of social networks and interactions within the networks play a significant role in influencing complexity of general practitioners that in turn influences their attitudes to medical care (responsibility for medical decisions). This is particularly useful for informing the design of the professional structure of general practitioners such that consideration is given for how the professional networks of GPs influence their involvement and responsibility for medical decision-making. At a practical level, the same theoretical framework and methodology can be applied beyond the general practitioner as an individual and to teams and organisations such as a hospital emergency department (ED). For instance, consider the utility of having a complexity dashboard that shows the extent to which the components of the ED (e.g., doctors, specialists, patients, bed resources, medical equipment, etc.) are interrelated or interdependent. By using the same complexity profiling methodology proposed in this paper, the complexity profiles can be associated with the operational performance of the ED, such as number of patients in the waiting list or the average waiting time for each patient prior to being treated in the ED. Once historical data is gathered about the association between complexity profiles and operational performance, a dashboard (likened to a fuel gauge in a car) would be a useful indicator to prevent poor operational performance and guide hospital policy (e.g., when the complexity profile is nearing the "relatively complex" level and this has been historically known to be associated with poor operational performance, then intervention in the use of ED resources would be required).

As with most research, there are a number of limitations. Firstly, the complexity framework developed here is based on relative, not absolute complexity that needs to be contextualized. In other words, the functional decomposition of the systems needs careful consideration. In this research, a simplified framework is proposed as an initial step to operationalize and demonstrate how aggregate complexity may be studied, characterized, and profiled, within the context of general practice. Secondly, in this work, professional relations pertaining to advice seeking and emotional closeness of actors were used. There is no allowance for the content of communication and this is obviously a drawback. Finally, it is also acknowledged that there may be other profiles of complexity between the four mentioned in this study. The boundaries of what constitutes a component to fall within the "simple" or "relatively complex," for example, also need further conceptual clarification. As this study is a preliminary attempt in characterizing aggregate complexity, it is contended that much research is needed in order to further our understanding of complexity-its conceptual definition and measures to operationalize it before one can even fathom its management and prediction.

To conclude, a number of avenues for moving this research forward are suggested. Firstly, one could possibly incorporate the intensity of complexity on a relational basis. That is, account for the intensity of complexity within the interaction itself. For example, a doctor may be categorized to be in the "simple" profile but their work might be extremely complex with a particular patient, compared to their other interactions with other components that are relatively mundane, simple, or routine. This impacts complexity on an aggregate level and must therefore be accounted for. Secondly, one could also compare other aspects of social network attributes with the complexity profiles. For instance, how would actors in a "simple" profile compare, in terms of network efficiency or constraint, against actors in a "complex" network? Network attributes such as efficiency and constraint are also linked to individual outcomes such as performance 
and this would be quite interesting. Thirdly, comparisons with other quadrants of complexity, for example, "relatively complex" versus "complex" and "complicated" versus "complex," could be carried out as well.

\section{Conflict of Interests}

The author declares that there is no conflict of interests regarding the publication of this paper.

\section{References}

[1] N. C. H. Stott and R. H. Davis, "The exceptional potential in each primary care consultation," The Journal of the Royal College of General Practitioners, vol. 29, no. 201, pp. 201-205, 1979.

[2] L. Gask and T. Usherwood, "ABC of psychological medicine: the consultation," British Medical Journal, vol. 324, no. 7353, pp. 1567-1569, 2002.

[3] A. Leader, C. Daskalakis, C. H. Braddock III et al., "Measuring informed decision making about prostate cancer screening in primary care," Medical Decision Making, vol. 32, no. 2, pp. 327336, 2012.

[4] K. S. K. Chung and L. Hossain, "Measuring performance of knowledge-intensive workgroups through social networks," Project Management Journal, vol. 40, no. 2, pp. 34-58, 2009.

[5] J. S. Coleman, E. Katz, and H. Menzel, "The diffusion of an innovation among physicians," Sociometry, vol. 20, no. 4, pp. 253-270, 1957.

[6] A. D. Innes, P. D. Campion, and F. E. Griffiths, "Complex consultations and the 'edge of chaos", British Journal of General Practice, vol. 55, no. 510, pp. 47-52, 2005.

[7] P. E. Plsek and T. Wilson, "Complexity, leadership, and management in healthcare organisations," British Medical Journal, vol. 323, no. 7315, pp. 746-749, 2001.

[8] R. L. Ackoff, “Towards a system of systems concepts,” Management Science, vol. 17, no. 11, pp. 661-671, 1971.

[9] Y. Bar-Yam, "Improving the effectiveness of health care and public health: a multiscale complex systems analysis," The American Journal of Public Health, vol. 96, no. 3, pp. 459-466, 2006.

[10] T. G. Kannampallil, G. F. Schauer, T. Cohen, and V. L. Patel, "Considering complexity in healthcare systems," Journal of Biomedical Informatics, vol. 44, no. 6, pp. 943-947, 2011.

[11] M. Mitchell, Complexity: A Guided Tour, Oxford University Press, New York, NY, USA, 2009.

[12] N. Johnson, Simply Complexity, Oneworld Publications, Oxford, UK, 2007.

[13] P. E. Plsek and T. Greenhalgh, "Complexity science: the challenge of complexity in health care," British Medical Journal, vol. 323, no. 7313, pp. 625-628, 2001.

[14] G. Bloom and S. Wolcott, "Building institutions for health and health systems in contexts of rapid change," Social Science \& Medicine, vol. 96, pp. 216-222, 2013.

[15] N. Keshavarz, D. Nutbeam, L. Rowling, and F. Khavarpour, "Schools as social complex adaptive systems: a new way to understand the challenges of introducing the health promoting schools concept," Social Science \& Medicine, vol. 70, no. 10, pp. 1467-1474, 2010.

[16] H. J. Lanham, L. K. Leykum, B. S. Taylor, C. J. McCannon, C. Lindberg, and R. T. Lester, "How complexity science can inform scale-up and spread in health care: understanding the role of self-organization in variation across local contexts," Social Science and Medicine, vol. 93, pp. 194-202, 2013.

[17] P. Nugus, K. Carroll, D. G. Hewett, A. Short, R. Forero, and J. Braithwaite, "Integrated care in the emergency department: a complex adaptive systems perspective," Social Science and Medicine, vol. 71, no. 11, pp. 1997-2004, 2010.

[18] Y. Bar-Yam, S. Bar-Yam, K. Z. Bertrand et al., "A complex systems science approach to healthcare costs and quality," NECSI Report, 2012.

[19] J. Horgan, "From complexity to perplexity," Scientific American, vol. 272, no. 6, pp. 104-109, 1995.

[20] S. M. Manson, "Simplifying complexity: a review of complexity theory," Geoforum, vol. 32, no. 3, pp. 405-414, 2001.

[21] S. P. Borgatti, A. Mehra, D. J. Brass, and G. Labianca, "Network analysis in the social sciences," Science, vol. 323, no. 5916, pp. 892-895, 2009.

[22] A.-L. Barabási, "Network science," Philosophical Transactions of the Royal Society A: Mathematical, Physical and Engineering Sciences, vol. 1987, pp. 1-3, 2013.

[23] S. Knoke and J. H. Kulinski, Network Analysis, Sage, Newbury Park, Calif, USA, 1992.

[24] J. O'Connor and I. McDermott, The Art of Systems Thinking, Thorsons, London, UK, 1997.

[25] M. Benham-Hutchins and T. R. Clancy, "Social networks as embedded complex adaptive systems," Journal of Nursing Administration, vol. 40, no. 9, pp. 352-356, 2010.

[26] D. Meltzer, J. Chung, P. Khalili et al., "Exploring the use of social network methods in designing healthcare quality improvement teams," Social Science \& Medicine, vol. 71, no. 6, pp. 1119-1130, 2010.

[27] E. West, D. N. Barron, J. Dowsett, and J. N. Newton, "Hierarchies and cliques in the social networks of health care professionals: implications for the design of dissemination strategies," Social Science and Medicine, vol. 48, no. 5, pp. 633-646, 1999.

[28] S. Wasserman and K. Faust, Social Network Analysis: Methods and Applications, Cambridge University Press, New York, NY, USA, 1994.

[29] M. S. Granovetter, "The strength of weak ties," The American Journal of Sociology, vol. 78, no. 6, pp. 1360-1380, 1973.

[30] D. Mascia and A. Cicchetti, "Physician social capital and the reported adoption of evidence-based medicine: exploring the role of structural holes," Social Science \& Medicine, vol. 72, no. 5, pp. 798-805, 2011.

[31] J. Scott, Social Network Analysis: A Handbook, Sage, London, UK, 2000.

[32] R. E. Mitchell and E. J. Trickett, "Task force report: social networks as mediators of social support. An analysis of the effects and determinants of social networks," Community Mental Health Journal, vol. 16, no. 1, pp. 27-44, 1980.

[33] U. Pfeil and P. Zaphiris, "Investigating social network patterns within an empathic online community for older people," Computers in Human Behavior, vol. 25, no. 5, pp. 1139-1155, 2009.

[34] J. Cockburn, D. Killer, E. Campbell, and R. W. Sanson-Fisher, "Measuring general practitioners' attitudes towards medical care," Family Practice, vol. 4, no. 3, pp. 192-199, 1987.

[35] C. Fullwood, A. Kennedy, A. Rogers et al., "Patients' experiences of shared decision making in primary care practices in the United Kingdom," Medical Decision Making, vol. 33, no. 1, pp. 26-36, 2013. 
[36] V. J. King, M. M. Davis, P. N. Gorman, J. B. Rugge, and L. J. Fagnan, "Perceptions of shared decision making and decision aids among rural primary care clinicians," Medical Decision Making, vol. 32, no. 4, pp. 636-644, 2012.

[37] C. B. Forrest, P. A. Nutting, S. von Schrader, C. Rohde, and B. Starfield, "Primary care physician specialty referral decision making: patient, physician, and health care system determinants," Decision Making in Clinical Practice, vol. 26, no. 1, pp. 76-85, 2006.

[38] R. M. Epstein, P. Franks, K. Fiscella et al., "Measuring patientcentered communication in Patient-Physician consultations: theoretical and practical issues," Social Science \& Medicine, vol. 61, no. 7, pp. 1516-1528, 2005.

[39] A. Mascarenhas and J. Norrie, Older Doctors Suffer the Shock of the New, The Sydney Morning Herald, Sydney, Australia, 2005.

[40] J. A. Jones, J. S. Humphreys, and M. A. Adena, Doctors' Perspective on the Viability of Rural Practice, 2004, http://pandora .nla.gov.au/pan/20865/20041115/rrh.deakin.edu.au/articles/ archiveview363d.html?ArticleID=305.

[41] R. P. Strasser, R. B. Hays, M. Kamien, and D. Carson, "Is Australian rural practice changing? Findings from the National Rural General Practice Study," The Australian Journal of Rural Health, vol. 8, no. 4, pp. 222-226, 2000.

[42] R. S. Burt, "Structural holes versus network closure," in Social Capital: Theory and Research, N. Lin, K. S. Cook, and R. S. Burt, Eds., Aldine de Gruyter, New York, NY, USA, 2000.

[43] J. S. Humphreys and F. Rolley, "A modified framework for rural general practice: the importance of recruitment and retention," Social Science and Medicine, vol. 46, no. 8, pp. 939-945, 1998.

[44] M. Mellow, "The work of rural professionals: doing the gemeinschaft-gesellschaft gavotte," Rural Sociology, vol. 70, no. 1, pp. 50-69, 2005.

[45] N. K. Choudhry, R. H. Fletcher, and S. B. Soumerai, "Systematic review: the relationship between clinical experience and quality of health care," Annals of Internal Medicine, vol. 142, no. 4, pp. 260-283, 2005.

[46] K. S. K. Chung, Understanding Attitudes to Performance in Knowledge-Intensive Work: The Influence of Social Networks and Information and Communication Technologies Use, Lambert Academic Publishing, Berlin, Germany, 2011.

[47] P. Marsden and K. E. Campbell, "Measuring tie strength," Social Forces, vol. 63, no. 2, pp. 482-501, 1984.

[48] H. R. Bernard, P. Killworth, D. Kronenfeld, and L. Sailer, "On the validity of retrospective data," Annual review of anthropology. Vol. 13, vol. 13, pp. 495-517, 1985.

[49] L. C. Freeman, A. K. Romney, and S. C. Freeman, "Cognitive structure and informant accuracy," American Anthropologist, vol. 89, no. 2, pp. 311-325, 1987.

[50] M. Hammer, "Explorations into the meaning of social network interview data," Social Networks, vol. 6, no. 4, pp. 341-371, 1984.

[51] C. M. McCarty and A. Wutich, "Conceptual and empirical arguments for including or excluding ego from structural analyses of personal networks," Connections, vol. 26, no. 2, pp. 82-88, 2005.

[52] R. S. Burt, Structural Holes: The Social Structure of Competition, Harvard University Press, Cambridge, Mass, USA, 1992. 


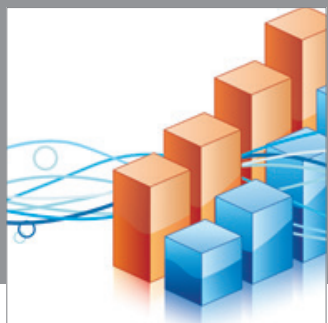

Advances in

Operations Research

mansans



The Scientific World Journal
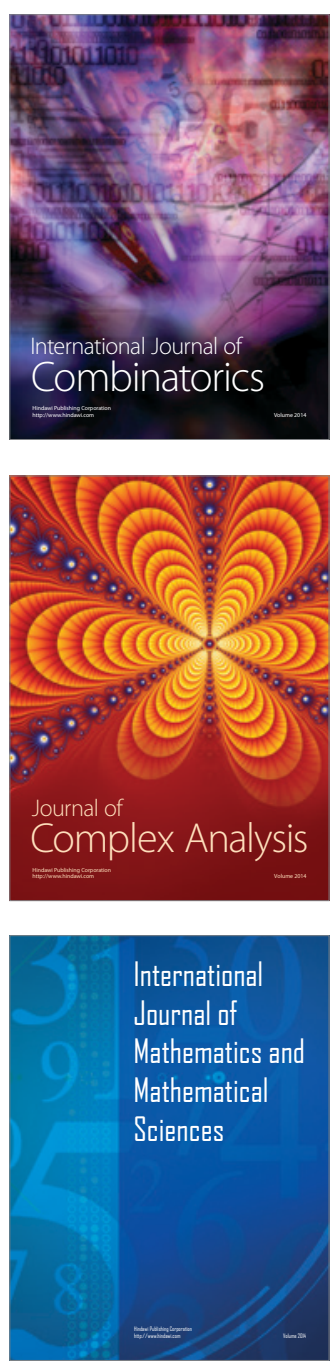
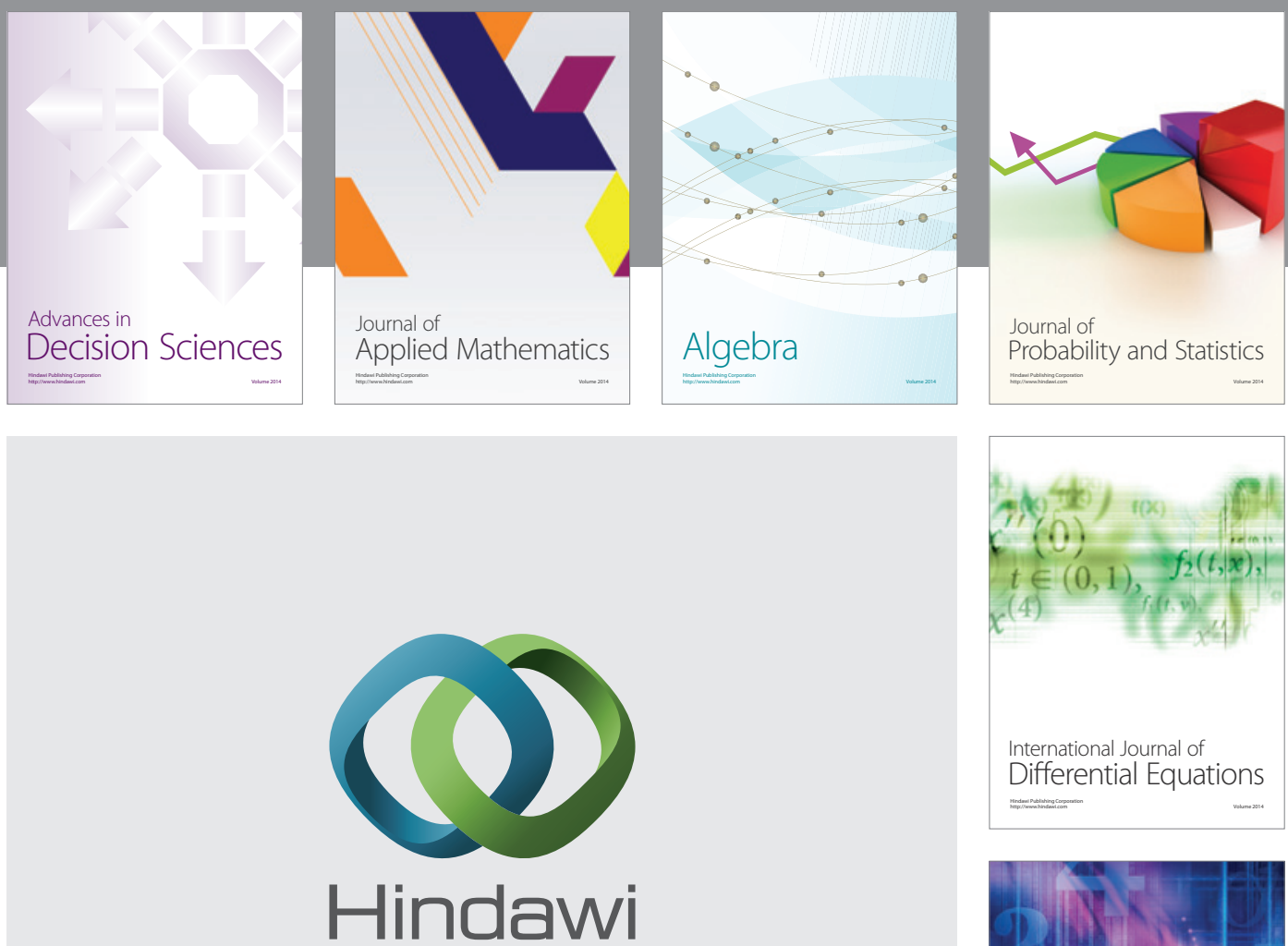

Submit your manuscripts at http://www.hindawi.com
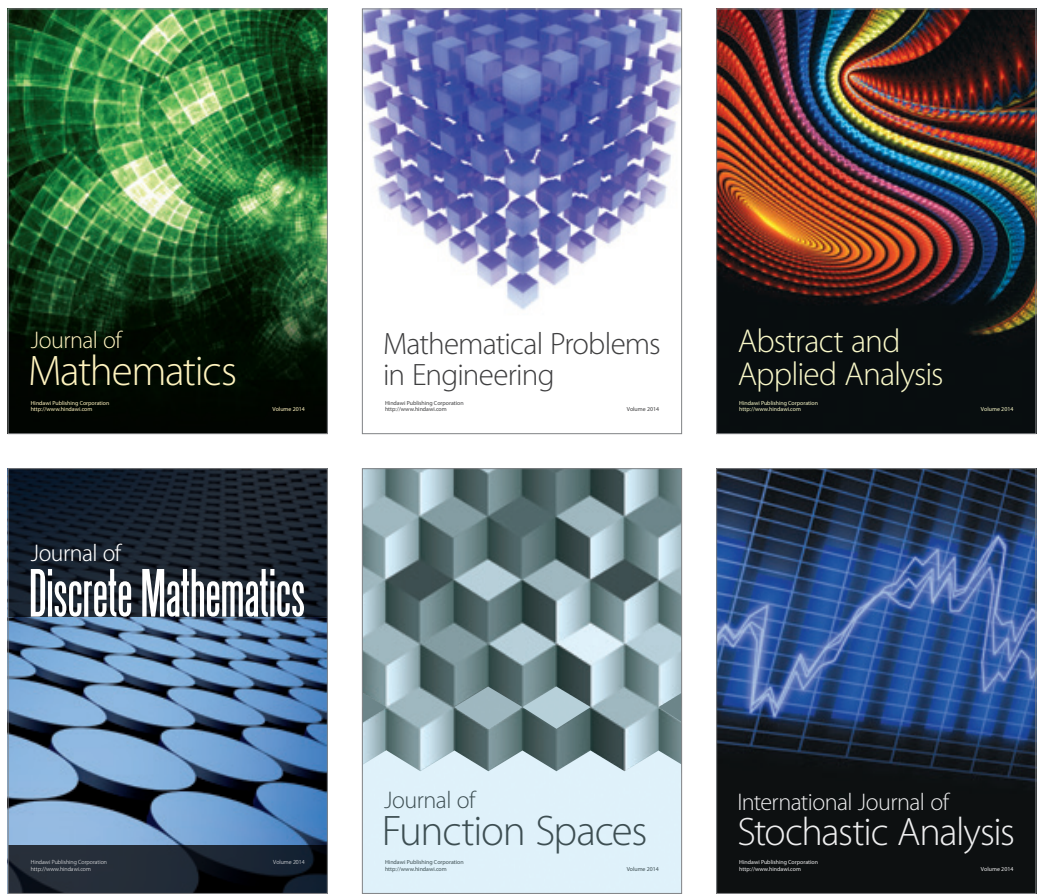

Journal of

Function Spaces



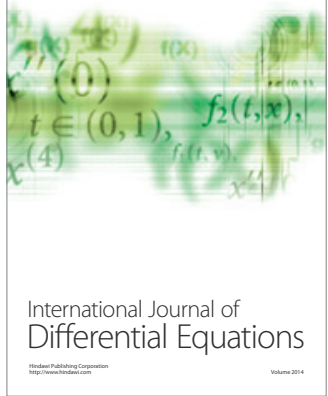
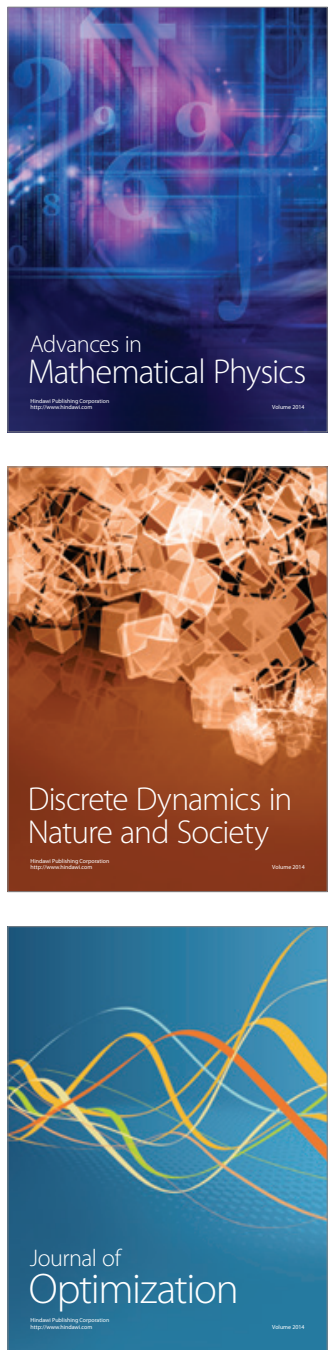